\title{
Analytische Beschreibung von Hot Disk-Sensoren zur Bestimmung von Methanolkonzentration und Strömungsgeschwindigkeit für Direktmethanolbrennstoffzellen
}

\author{
Bastian Schmitt, Andreas Schütze \\ Lehrstuhl für Messtechnik, Universität des Saarlandes, Saarbrücken \\ Tel.: 0681/302-5017, e-mail: b.schmitt@Imt.uni-saarland.de
}

\section{Kurzfassung}

Die analytische Beschreibung eines neu entwickelten Sensor zur Messung binärer Mischungsverhältnisse basierend auf dem sog. Hot Disk-Verfahren wird vorgestellt. Basierend auf der Betrachtung des Heizers als punktförmige Quelle oder als Quelle mit einer bzgl. ihres Mittelpunkts Gauß-förmigen Verteilung der Flächenheizleistungsdichte kann die gemessene Temperaturerhöhung adäquat beschrieben werden. Zusätzlich wird gezeigt, warum im vorliegenden Fall bei einer auftretenden Strömung durch Platzierung eines Temperatursensors hinter dem Heizer und Nutzung der Pulsdrahtanemometrie zwischen Heizer und Sensor die Strömungsgeschwindigkeit unabhängig von den thermischen Eigenschaften des Gemischs bestimmt werden kann.

\section{Einleitung}

Die Kontrolle des Mischungsverhältnisses binärer Gemische wird in vielen technischen Systemen für die optimale Prozessführung und -überwachung benötigt. Wichtige Einsatzbereiche reichen von der Bestimmung des Methanolanteils in Wasser für Direktmethanolbrennstoffzellen (engl. Direct Methanol Fuel Cell, DMFC) über Harnstoff in Wasser für die Abgasnachbehandlung von Dieselfahrzeugen mittels selektiver katalytischer Reduktion bis hin zur Bestimmung des Wassergehalts in der Bremsflüssigkeit. Gerade bei dynamisch betrie- 
benen DMFCs wird ein Sensor benötigt, um den von der Brennstoffzelle im Betrieb verbrauchten Anteil an Methanol nachdosieren zu können und so den optimalen Arbeitspunkt von je nach Zelltyp etwa 1,2 bis 4,1 Vol.-\% Methanol und damit eine lange Laufzeit zu gewährleisten [1]. Zusätzlich zur genauen und stabilen Messung spielen hierbei geringe Kosten und eine niedrige Leistungsaufnahme für mobile Anwendungen wie Laptops eine wichtige Rolle.

\section{Messprinzip}

Das untersuchte, thermische Sensorprinzip basiert auf den transienten, zur Charakterisierung von Feststoffen bekannten Flächenheizertechniken (engl. transient plane source techniques), die sich je nach Form des Heizers in hot strip oder hot disk-Methoden einteilen lassen [2]. Es macht sich die unterschiedlichen thermischen Eigenschaften der Komponenten in der Mischung zunutze, wobei die Ausbreitung eines eingeprägten Heizpulses sowohl zeitlich als auch örtlich bestimmt wird. Das Layout des genutzten, mikrostrukturierten Sensors ist in Bild 1 gezeigt. Es besteht aus einem zentralen Heizer, der in unterschiedlichen Abständen von vier Temperatursensoren umgeben ist. Im Betrieb wird der Heizer mit einem Heizpuls beaufschlagt und sowohl seine als auch die Temperaturerhöhung der vier Sensoren gemessen, um daraus bei bekannten Komponenten in der Mischung das Mischungsverhältnis und die Strömungsgeschwindigkeit zu bestimmen. Realisiert wurden die Sensoren durch Sputtern einer ca. $500 \mathrm{~nm}$

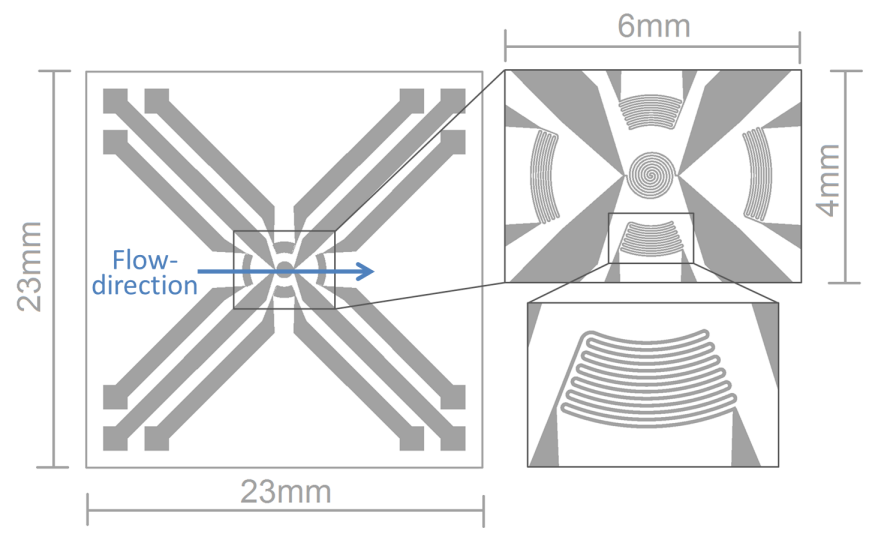

Bild 1: Layout der untersuchten, mikrostrukturierten Sensoren mit Detailansicht des zentralen Heizers und der vier Temperatursensoren (nach [3]). Der Pfeil symbolisiert die Strömungsrichtung. 
Aluminiumschicht auf eine $125 \mu \mathrm{m}$ dicke Kapton HN-Folie (Fa. DuPont) und Passivieren der Strukturen mit einem Polyamidimid-Lack mit ca. $5 \mu \mathrm{m}$ Dicke. Der Aufbau und die Herstellung sind in [3] genauer beschrieben.

\section{Mathematische Beschreibung des Messprinzips}

Unter Vernachlässigung der Temperaturabhängigkeit der Stoffeigenschaften lautet die Wärmeleitungsgleichung in einem ruhenden, isotropen und inkompressiblen Medium [4]:

$$
\alpha \nabla^{2} T(\boldsymbol{x}, t)+\frac{Q(\boldsymbol{x}, t)}{\rho c_{p}}=\frac{\partial T(\boldsymbol{x}, t)}{\partial t}
$$

Hierbei bezeichnet $\alpha=\lambda /\left(\rho c_{p}\right)$ die Temperaturleitfähigkeit mit der Wärmeleitfähigkeit $\lambda$, der Dichte $\rho$ und der spezifischen Wärmekapazität $c_{p}, \nabla^{2}$ steht für den Laplace-Operator mit $\nabla^{2}=\nabla \cdot \nabla, T(\boldsymbol{x}, t)$ für die Temperatur am Ort $\boldsymbol{x}=[x, y, z]$ zur Zeit $t$ und $Q$ die pro Volumen eingeprägte Leistung. Unter der Annahme $Q(\boldsymbol{x}, t)=0$ lautet die Fundamentallösung $G(\boldsymbol{x}, t)$ zu Gl. (1) mit den Anfangs- und Randbedingungen $T(\boldsymbol{x}, 0)=0$ und $T(\infty, 0)=0$ [5]

$$
G(\boldsymbol{x}, t)=\frac{1}{(4 \pi \alpha t)^{3 / 2}} \exp \left(-\frac{\|\boldsymbol{x}\|^{2}}{4 \alpha t}\right),
$$

wobei $\|\boldsymbol{x}\|$ für die Euklidische Norm von $\boldsymbol{x}$ steht. Im allgemeinen Fall mit der Anfangstemperatur $T(\boldsymbol{x}, 0)=T_{0}$ und $Q(\boldsymbol{x}, t) \neq 0$ ergibt sich die Lösung der Wärmeleitungsgleichung durch Faltung der Anfangstemperatur und der Quellenverteilung $Q(\boldsymbol{x}, t) /\left(\rho c_{p}\right)$ mit Gl. (2) [6], wodurch der Temperaturanstieg $\Delta T(\boldsymbol{x}, t)=T(\boldsymbol{x}, t)-T_{0}$ folgt zu:

$$
\Delta T(\boldsymbol{x}, t)=\int_{0}^{t} \int_{V^{\prime}} \frac{Q\left(\boldsymbol{x}^{\prime}, t^{\prime}\right)}{\rho c_{p}} \frac{1}{\left(4 \pi \alpha\left(t-t^{\prime}\right)\right)^{3 / 2}} \exp \left(-\frac{\left\|\boldsymbol{x}-\boldsymbol{x}^{\prime}\right\|^{2}}{4 \alpha\left(t-t^{\prime}\right)}\right) \mathrm{d}^{3} \boldsymbol{x}^{\prime} \mathrm{d} t^{\prime}
$$

Zur analytischen Betrachtung der Temperaturausbreitung aufgrund eines Heizpulses der gefertigten, mikrothermischen Heizer werden im Folgenden die zwei Spezialfälle untersucht, in denen der Heizer als Punktquelle betrachtet wird bzw. eine bzgl. des Zentrums des Heizers Gauß-förmige Verteilung der Flächenheizleistungsdichte vorliegt. Diese kann im vorliegenden Fall als Näherung genutzt werden, da sich aufgrund des gewählten, runden Heizerlayouts nach einer 
gewissen Heizdauer eine nahezu Gauß-förmige Temperaturverteilung ergibt. Bild 2 zeigt einen Querschnitt der unter Nutzung von Comsol Multiphysics (Fa. Comsol AB) simulierten Temperaturverteilung eines mit $30 \mathrm{~mW}$ beaufschlagten Heizers mit $0,5 \mathrm{~mm}$ Durchmesser in Wasser sowie die mit einer Normalverteilung gefittete Temperaturverteilung nach einer Heizdauer von 0,3 s. Aufgrund der Proportionalität der lokalen Heizleistung zum lokalen Heizwiderstand bzw. zur lokalen Heizertemperatur kann somit näherungsweise nach einer gewissen Heizdauer auch die Verteilung der Flächenheizleistungsdichte als bzgl. der Heizermitte normalverteilt angenommen werden.

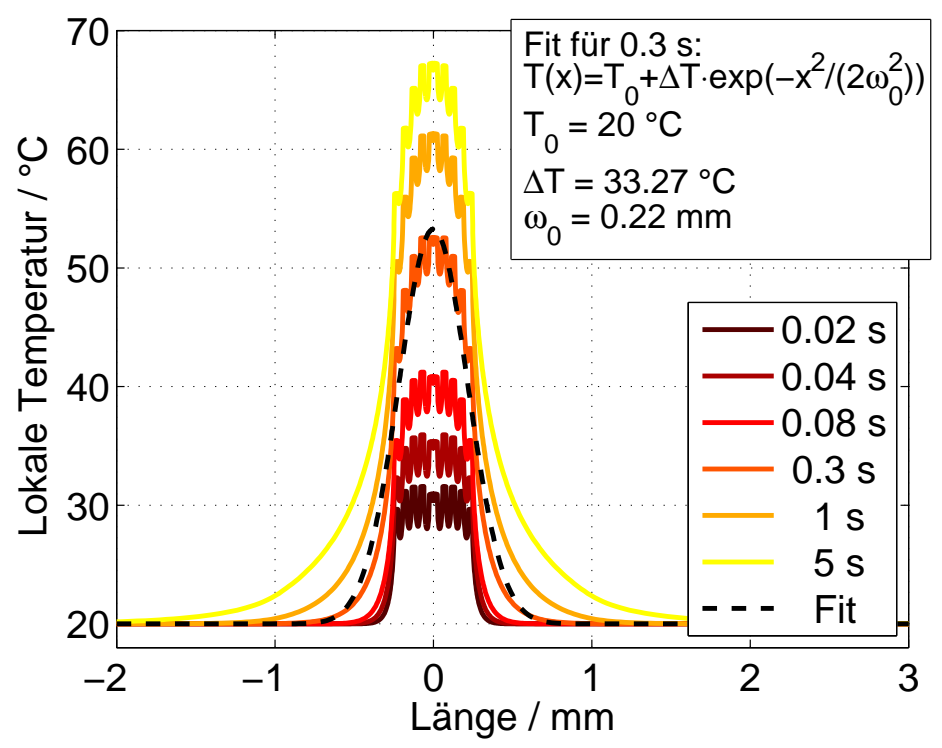

Bild 2: Simulierte Temperaturverteilung eines mit $30 \mathrm{~mW}$ beaufschlagten Heizers mit 0,5 mm Durchmesser in Wasser nach verschiedenen Heizdauern.

\subsection{Punktförmige Wärmequelle}

Zunächst soll die Wärmeausbreitung unter Annahme einer vernachlässigbaren Ausdehnung des Heizers gegenüber dem zu messenden Flüssigkeitsgemisch untersucht werden, wobei der als punktförmig angenommene Heizer in dieser Darstellung vollständig vom Gemisch umgeben ist. Für diese im Ursprung $\boldsymbol{x}=0$ zum Zeitpunkt $t=0$ eingeschalteten Punktquelle $Q_{p}$ mit konstanter Leistung $P_{P}$ (siehe Gl. (4)), wobei $\delta(x)$ die Dirac-Funktion und $\Theta(t)$ die Heaviside-Funk-

$$
Q_{P}(\mathbf{0}, t)=P_{P} \delta(x) \delta(y) \delta(z) \Theta(t)
$$


tion repräsentieren, ergibt sich die Temperatur im Abstand $\|\boldsymbol{x}\|$ zur Quelle durch Einsetzen in Gl. (3) und Integration über den ganzen Raum nach [6] zu

$$
\Delta T(\boldsymbol{x}, t)=\frac{P_{P}}{\rho c_{p}} \frac{1}{4 \pi \alpha} \frac{1}{\|\boldsymbol{x}\|} \operatorname{erfc}\left(\frac{\|\boldsymbol{x}\|}{\sqrt{4 \alpha t}}\right)=\frac{P_{P}}{\lambda} \frac{1}{4 \pi} \frac{1}{\|\boldsymbol{x}\|} \operatorname{erfc}\left(\frac{\|\boldsymbol{x}\|}{\sqrt{4 \alpha t}}\right),
$$

mit dem komplementären Fehlerintegral erfc(·) [7]. Mit Gl. (5) kann die Temperatur auf der Oberfläche des Heizers bei $\boldsymbol{x} \rightarrow 0$ nicht bestimmt werden, da der Ausdruck divergiert. Allerdings wird $\operatorname{erfc}(x)$ für Argumente von $x>1,5$ vernachlässigbar, wodurch sich Gl. (5) der Einfachheit halber zur Abschätzung der Laufzeit $t_{\max }$ eines Heizpulses zum Erreichen einer Grenzfläche in gegebener Entfernung $\boldsymbol{x}$ bzw. zur Abschätzung der Eindringtiefe $h_{\max }$ des Wärmepulses in das Gemisch nach einer vorgegebenen Heizdauer $t_{\max }$ eignet. Hierbei folgt der Zusammenhang

$$
t_{\text {max }} \approx h_{\text {max }}^{2} /(9 \alpha)
$$

Die Temperaturleitfähigkeit verringert sich in dem für DMFCs interessanten Konzentrationsbereich von 0 bis 10 Vol.-\% Methanol in Wasser von ca. 1,41 auf $1,29 \times 10^{-7} \mathrm{~m}^{2} / \mathrm{s}$ [8]. Bei gestoppter Strömung kann die Laufzeit eines Temperaturpulses vom Heizer zu einem $2 \mathrm{~mm}$ hinter dem Heizer befindlichen Temperatursensor aus Gl. (6) für Wasser zu 3,15 s abgeschätzt werden und steigt bei einer Konzentration von 10 Vol.-\% Methanol auf 3,45 s an. Die mittleren Geschwindigkeiten ergeben sich somit zu 0,63 bzw. $0,58 \mathrm{~mm} / \mathrm{s}$, wodurch der Einfluss des Methanols maximal 0,05 mm/s oder ca. 8,3\% beträgt.

Liegt eine Strömung über dem Heizer vor, so wird zusätzlich Wärme durch erzwungene Konvektion abgeführt, die sich mit der Wärmeausbreitung aufgrund der Wärmeleitung überlagert. Für die für DMFCs interessanten Flussraten zwischen 50 und $200 \mathrm{ml} / \mathrm{h}$ ergeben sich aufgrund des gewählten Kammerquerschnitts von $8 \times 1 \mathrm{~mm}^{2}$ [3] mittlere Strömungsgeschwindigkeiten von ca. 1,74 bis $6,94 \mathrm{~mm} / \mathrm{s}$. Der Einfluss des Methanols beträgt somit bei einer hohen Flussrate von $200 \mathrm{ml} / \mathrm{h}$ nur ca. $0,05 /(6,94+0,6)=0,7 \%$ und erhöht sich bei einer geringen Flussrate von $50 \mathrm{ml} / \mathrm{h}$ auf 2,1\%. Dies ermöglicht die Abschätzung des Messfehlers der Messung der Strömungsgeschwindigkeit mittels der Laufzeitmethode bei Unkenntnis der aktuellen Methanolkonzentration. 


\subsection{Gauß-förmige Wärmequelle}

Unter Annahme einer rotationssymmetrischen, in der xy-Ebene liegenden und zum Zeitpunkt $t=0$ eingeschalteten Quelle $Q_{G}$ mit einer bzgl. ihrem Zentrum normalverteilten Flächenheizleistungsdichte lässt sich diese nach [9] mathematisch beschreiben als

$$
Q_{G}(x, y, z, t)=\frac{P_{G}}{2 \pi \omega_{0}^{2}} \exp \left(-\frac{x^{2}+y^{2}}{2 \omega_{0}^{2}}\right) \delta(z) \Theta(t),
$$

wobei $P_{G}$ die eingeprägte Heizleistung darstellt und $\omega_{0}$ für die Standardabweichung steht. Ohne Beachtung des zeitlichen Verlaufs ergibt die Integration der Leistungsverteilung über den gesamten Raum die insgesamt eingeprägte Heizleistung $P_{G}$. Durch Fitten der in Finite-Elemente Simulationen unter Nutzung von Comsol Multiphysics erhaltenen Temperaturverteilung des Heizers entspricht $\omega_{0}$ in dieser Darstellung in etwa dem Radius des Heizers (siehe Bild 2), wodurch sich die erwartete Temperaturerhöhung später aus der analytischen Lösung leicht abschätzen lässt. Durch Einsetzen von Gl. (7) in Gl. (3) und unter Annahme einer zeitlich konstanten Heizleistung ergibt sich die Temperaturerhöhung in der Heizerebene bei $z=0$ durch Integration über den gesamten Raum [6] unter Einführung des Wärmeeindringkoeffizienten $b=\sqrt{\lambda \rho c_{p}} \mathrm{zu}$

$$
\Delta T(\boldsymbol{x}, t)=\frac{P_{G}}{4 \pi^{3 / 2} b} \int_{t^{\prime}=0}^{t} \frac{1}{\sqrt{t-t^{\prime}}} \frac{1}{2 \alpha\left(t-t^{\prime}\right)+\omega_{0}^{2}} \exp \left(-\frac{x^{2}+y^{2}}{4 \alpha\left(t-t^{\prime}\right)+2 \omega_{0}^{2}}\right) \mathrm{d} t^{\prime},
$$

was sich im Mittelpunkt des Heizers bei $\boldsymbol{x}=0$ vereinfacht zu [6]

$$
\Delta T(\mathbf{0}, t)=\frac{P_{G}}{(2 \pi)^{3 / 2} \lambda \omega_{0}} \arctan \left(\sqrt{\frac{2 \alpha t}{\omega_{0}^{2}}}\right) .
$$

Diese Funktion kann unmittelbar zum Fitten des Temperaturanstiegs des Heizers genutzt werden. Die Funktion $\arctan (x)$ lässt sich für kleine Argumente $x<0,25$ mit einem relativen Fehler kleiner als $2 \%$ durch das Argument ersetzen. Somit ergibt sich

$$
\Delta T(\mathbf{0}, t) \approx \frac{P_{G}}{(2 \pi)^{3 / 2} \lambda \omega_{0}} \sqrt{\frac{2 \alpha t}{\omega_{0}^{2}}}=\frac{P_{G}}{2 \pi^{3 / 2} \omega_{0}^{2}} \frac{1}{b} \sqrt{t},
$$


wodurch bei kurzen Heizdauern ein wurzelförmiger Anstieg der Heizertemperatur erwartet wird, der sich auch bei Annahme einer Flächenquelle ergeben würde (vgl. Gl. (4.39) in [6] für $z=0$ ). Für sehr große Argumente konvergiert $\arctan (\cdot)$ gegen $\pi / 2$, wodurch sich die nach einer langen Heizdauer erwartete Temperaturerhöhung aus Gl. (9) abschätzen lässt zu

$$
\lim _{t \rightarrow+\infty} \Delta T(\mathbf{0}, t)=\frac{P_{G}}{4 \sqrt{2 \pi} \lambda \omega_{0}} .
$$

Da messtechnisch durch Messung der (mittleren) Heizer- und Sensorwiderstände ebenfalls nur die mittleren Temperaturen $\bar{T}_{H}$ des Heizers bzw. $\bar{T}_{S}$ des Sensors messbar sind, müssen diese noch aus Gl. (8) bestimmt werden. Dies erfolgt durch Nutzung von Polarkoordinaten und Ersetzen von $r^{2}:=x^{2}+y^{2}$ unter Anwendung des Mittelwertsatzes der Integralrechnung [10] durch Integration der Temperaturverteilung über die betrachtete Fläche. Für den Heizer in der Ebene $z=0$ mit Radius $r_{h}=\omega_{0}$ ergibt sich die mittlere Temperatur durch Integration über $r$ von 0 bis $\omega_{0}$ und über $\phi$ von 0 bis $2 \pi \mathrm{zu}$

$$
\begin{aligned}
\bar{T}_{H}-T_{0}=\frac{P_{G}}{4 \pi^{3 / 2} b} \frac{1}{\pi \omega_{0}^{2}} \int_{t^{\prime}=0}^{t} \int_{\phi=0}^{2 \pi} \int_{r=0}^{\omega_{0}} \frac{1}{\sqrt{t-t^{\prime}}} \frac{1}{2 \alpha\left(t-t^{\prime}\right)+\omega_{0}^{2}} \\
\quad \exp \left(-\frac{r^{2}}{4 \alpha\left(t-t^{\prime}\right)+2 \omega_{0}^{2}}\right) r \mathrm{~d} r \mathrm{~d} \phi \mathrm{d} t^{\prime},
\end{aligned}
$$

was näherungsweise dem 0,84-fachen der Temperatur in der Heizermitte (siehe Gl. (9)) entspricht. Analog kann die Temperaturerhöhung eines neben dem Heizer befindlichen Temperatursensors bestimmt werden, wobei die Integrationsgrenzen von $r$ auf den Abstand des Sensors anzupassen sind und $\phi$ beim gewählten Layout einen Winkel von $45^{\circ}(=\pi / 4)$ überstreicht.

Zuletzt muss beachtet werden, dass die durch den Heizer eingeprägte Energie nur zum Teil durch das Gemisch abgeführt wird und zum Teil in das Grundsubstrat abfließt. Da der Temperaturverlauf an der Grenzfläche zwischen Substrat und Gemisch stetig ist, gilt, dass die Temperatur in einem infinitesimalen Abstand $\pm \delta z$ von der Grenzfläche im Gemisch und im Substrat gleich sein muss. Für kurze Heizdauern folgt damit aus Gl. (10), dass für die dem Gemisch zu- 
geführte Heizleistung $P_{G, F}$ gilt:

$$
\Delta T(0,0, \delta z, t) \stackrel{!}{=} \Delta T(0,0,-\delta z, t) \Rightarrow P_{G, F}=\frac{P_{G}}{1+b_{s} / b_{g}},
$$

mit $b_{s}$ und $b_{g}$ den Wärmeeindringkoeffizienten des Grundsubstrats bzw. des Gemischs. Da die hiermit berechnete Heizleistung schließlich nur noch in einen Halbraum abgeführt wird, die vorherigen Herleitungen allerdings für den gesamten Raum gelten, muss der abgeschätzte Temperaturanstieg noch mit dem Faktor 2 multipliziert werden. Aufgrund des im vorliegenden Falle gewählten Messaufbaus mit einer Bohrung unter dem Polyimidsubstrat zur thermischen Entkopplung des Heizers [3] und des vernachlässigbaren Wärmeeindringkoeffizienten von Luft kann der Wärmeeintrag hier als vollständig in das zu vermessende Flüssigkeitsgemisch abfließend betrachtet werden.

\section{Ergebnisse}

Bild 3 zeigt den gemessenen Temperaturanstieg des zentralen Heizers in Stopped-Flow Messungen in einem für DMFCs typischen Konzentrationsbereich von Methanol in Wasser sowohl in linearer als auch doppeltlogarithmischer Darstellung, um den bei kurzen Heizdauern erhaltenen, wurzelförmigen Anstieg zu belegen. Zusätzlich ist der mit dem hergeleiteten Modell unter Annahme einer normalverteilten Flächenheizleistungsdichte erwartete Temperaturanstieg für die in der Literatur bzw. in Datenblättern verfügbaren Stoffeigenschaften dargestellt, der sowohl die Form des Temperaturverlaufs als auch die auftretenden Temperaturen angemessen beschreibt, wobei der Wärmeabfluss in die Folie aufgrund ihrer geringen Dicke und der darunter befindlichen Luftschicht mit ihrem vernachlässigbaren Wärmeeindringkoeffizienten vernachlässigt wurde. Unter Nutzung des vereinfachten Modells für den Heizermittelpunkt (siehe Gl. (9)) ergibt sich hierbei in Wasser ein erwarteter Temperaturanstieg des Heizermittelpunkts $\bar{T}_{H, M}(t)$, der der Funktion $\bar{T}_{H, M}(t)=20,6+29,9 \cdot \operatorname{atan}(2,4 \sqrt{x})$ folgt und mit den Koeffizienten des Fits in Bild 3 links vergleichbar ist, wobei hierfür die Anfangstemperatur übernommen wurde. Der Temperaturanstieg der Temperatursensoren hinter dem Heizer ist in Bild 4 für verschiedene Methanolkonzentration und Flussraten dargestellt. 

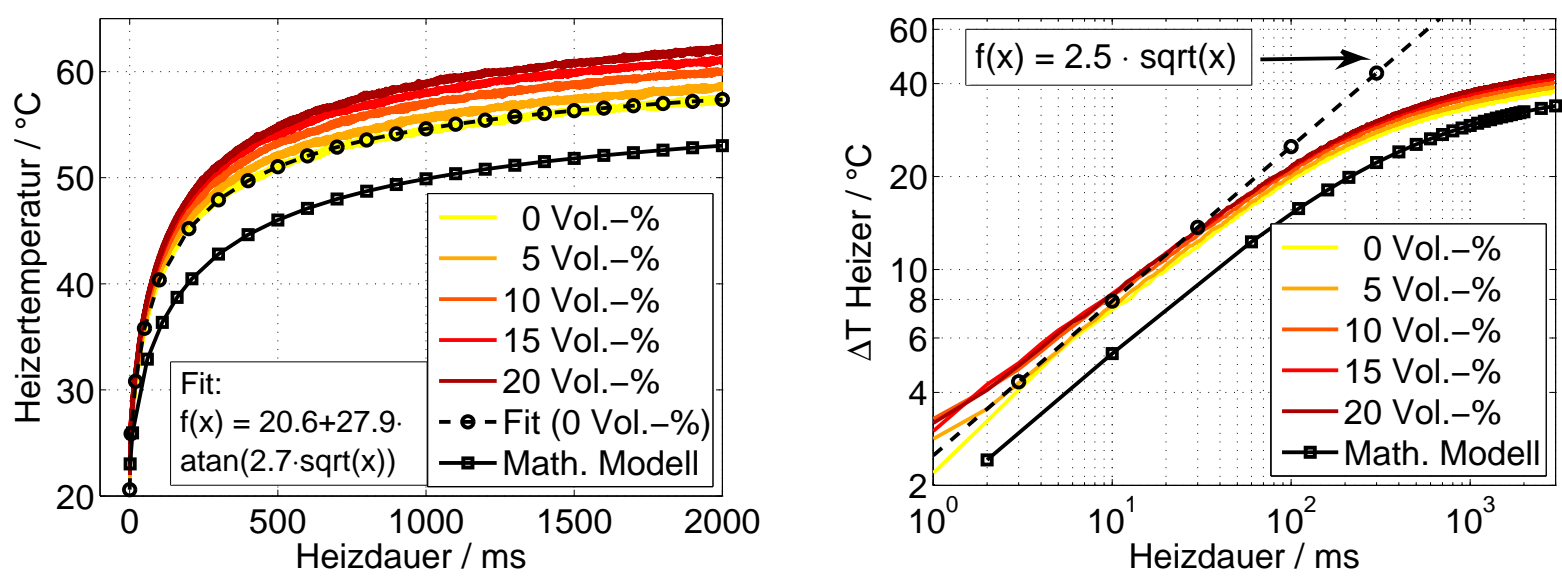

Bild 3: Lineare und doppelt-logarithmische Darstellung des Temperaturanstiegs des zentralen Heizers in Stopped-Flow-Messungen für Methanol in Wasser (nach [3]) mit Fit des Anstiegs und Darstellung des erwarteten Verlaufs in Wasser unter Nutzung des hergeleiteten, mathematischen Modells.

Ohne vorliegende Strömung zeigt das hergeleitete, mathematische Modell eine gute Approximation des gemessenen Verlaufs, wobei die gemessene, höhere Temperatur unter anderem auf die den Heizer bedeckende Passivierung zurückzuführen ist. Bei höheren Flussraten ist der Temperaturanstieg wie zuvor herge-

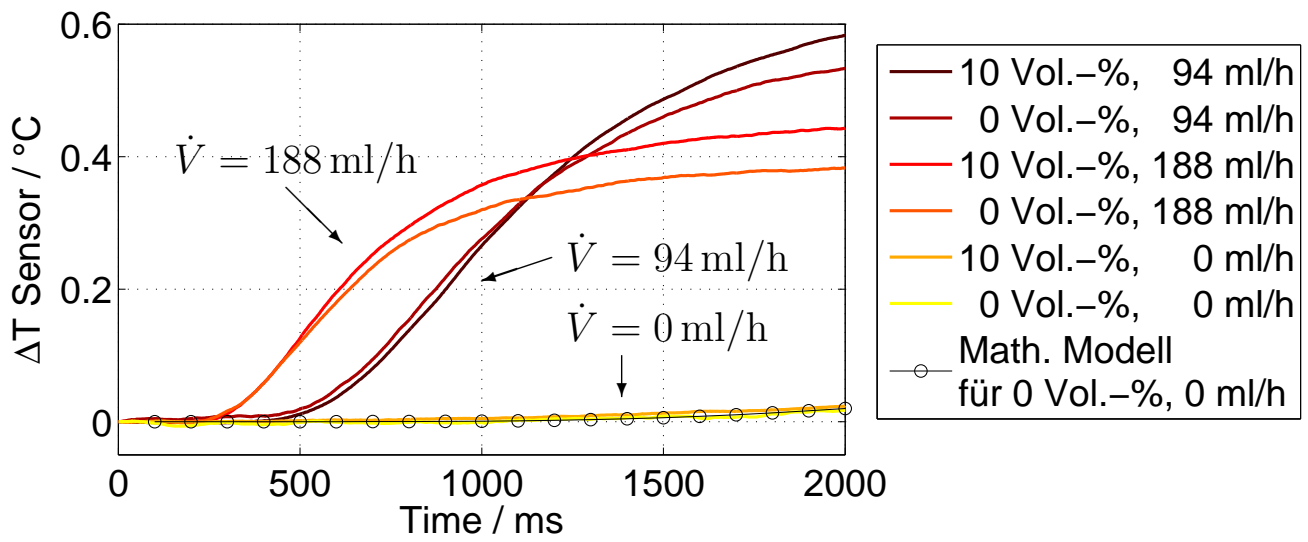

Bild 4: Gemessener Temperaturanstiegs des Sensors in Strömungsrichtung $2 \mathrm{~mm}$ hinter dem Heizer für verschiedene Flussraten $\dot{V}[\mathrm{ml} / \mathrm{h}]$ und Methanolkonzentrationen $\phi$ [Vol.-\%] (nach [3]).

leitet zunächst unabhängig von der Methanolkonzentration und ermöglicht die Bestimmung der Strömungsgeschwindigkeit unabhängig von der Methanolkonzentration [3]. Basierend auf ihrer Kenntnis soll der Einfluss der erzwungenen Konvektion auf die Temperaturerhöhung des Heizers kompensiert werden. 


\section{Fazit}

Die entwickelten Hot Disk-Sensoren zur Bestimmung von Flüssigkeitsgemischen konnten mathematisch beschrieben werden. Das entwickelte Modell beschreibt sowohl den bei Methanol/Wasser-Gemischen für Direktmethanolbrennstoffzellen gemessenen Temperaturanstieg als auch die Möglichkeit der simultanen Messung der Strömungsgeschwindigkeit unter Nutzung des Prinzips der Pulsdrahtanemometrie und ermöglicht die Optimierung des Messprinzips.

\section{Literatur}

[1] Nölke, M.: „Entwicklung eines Direkt-Methanol-Brennstoffzellensystems der Leistungsklasse kleiner 5kW“, Forschungszentrum Jülich, Jülich, 2007

[2] Gustafsson, S. E.: "Transient plane source techniques for thermal conductivity and thermal diffusivity measurements of solid materials", Review of Scientific Instruments 62 (3), pp. 797-804, 1991, DOI: 10.1063/1.1142087

[3] Schmitt, B.; Kiefer, C.; Schütze, A.: "Microthermal sensors for determining fluid composition and flow rate in fluidic systems“, Microsystem Technologies 20 (4-5), pp. 641-652, 2014, DOI: 10.1007/s00542-013-2001-y

[4] H. K. Carslaw, J. C. Jaeger: "Conduction of heat in solids", Oak Ridge National Laboratory, Vol. 985, 1959

[5] Y.He: "Rapid thermal conductivity measurement with a hot disk sensor Part 1. Theoretical considerations“, Thermochimica Acta, Vol. 436, No. 12, pp. 122-129, 2005, DOI 10.1016/j.tca.2005.06.026

[6] R. Poprawe: „Lasertechnik für die Fertigung“, Springer-Verlag Berlin Heidelberg, 2005

[7] H.D. Baehr, K. Stephan: „Wärme- und Stoffübertragung“, 8. Auflage, Springer-Verlag Berlin Heidelberg, 2013

[8] J. Wang, M. Fiebig: "Measurement of the thermal diffusivity of aqueous solutions of alcohols by a laser-induced thermal grating technique", International Journal of Thermophysics 16 (6), pp. 1353-1361, 1995, DOI: 10.1007/BF02083545

[9] H. Gatignon: "Statistical Analysis of Management Data", Springer New York Heidelberg Dordrecht London, 3rd ed., 2014

[10] O. Forster: „Analysis 1“, Vieweg+Teubner, 9., überarbeitete Auflage, 2008 